\title{
Total ankylosis of the bilateral hand and wrist joints in a patient with ankylosing spondylitis
}

Shin-Gil Kim and Kyung-Ann Lee

Division of Rheumatology, Department of Internal Medicine, Soonchunhyang University Seoul Hospital, Seoul, Korea
Received: March 18, 2020

Revised : March 20, 2020

Accepted: March 20, 2020

\section{Correspondence to}

Kyung-Ann Lee, M.D.

Tel: +82-2-710-3063

Fax: +82-2-709-9554

E-mail: cyberag@naver.com https://orcid.org/0000-00017499-6363
A 64-year-old man was referred to our rheumatology clinic for preoperative assessment prior to cataract surgery. He was diagnosed with ankylosing spondylitis (AS) 16 years earlier; however, treatment was discontinued owing to nonsteroidal anti-inflammatory drug-induced gastric ulcer bleeding 10 years ago. He had persistent back pain with stiffness and stayed at home in a bedridden state for several years. On physical examination, both hip joints were fixed in the flexion state, and both hand and wrist joints had severely limited motions. Laboratory examination revealed the following: C-reactive protein level, $0.90 \mathrm{mg} / \mathrm{dL}$ (normal range, 0.0 to 0.5 ); erythrocyte sedimentation rate, $120 \mathrm{~mm} / \mathrm{hr}$ (normal range, o to 20); negative findings for the rheumatoid factor and anti-citrullinated protein antibody; and positive findings for human leukocyte antigen-B27. Radiography showed bamboo spine and ankylosis of the sacroiliac and hip joints (Fig. 1). Total ankylosis in most hand and wrist joints is shown in Fig. 2.

Arthritis in the peripheral joint is found in $30 \%$ to $50 \%$ of patients with AS, which can occur at any point in the disease course. Peripheral arthritis in AS predominantly presents in the lower limbs, usually in an asymmetrical fashion. Symmetric total ankylosis of the hand and wrist joints is extremely rare in AS. Contrary to rheumatoid arthritis, our case showed involvement of the distal interphalangeal joints in combination with other finger and wrist joints. Herein, we report that AS could affect the small joints of the hand and wrist symmetrically, resulting in extensive deformities with severe disability. Optimal treatment and disease monitoring of patients with AS are essential to reduce inflammation and prevent functional limitations.

This study was conducted in accordance with the Declaration of Hel-

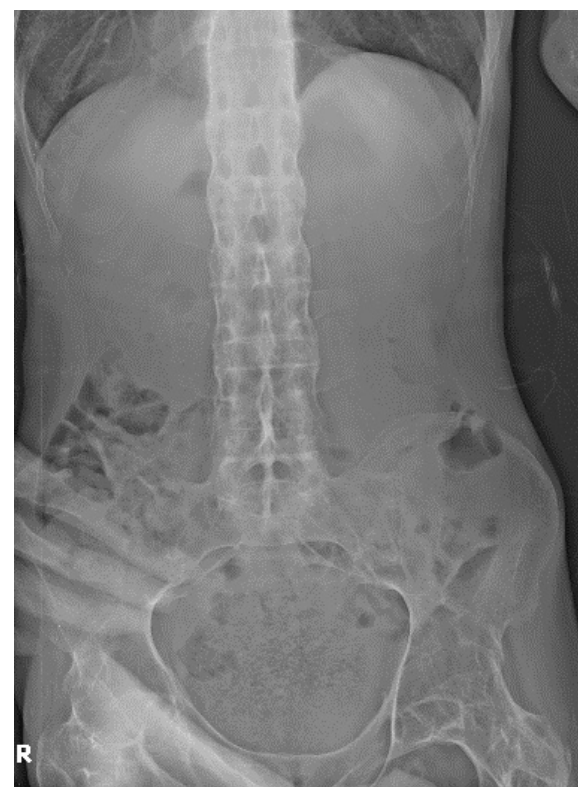

Figure 1. Plain lumbar radiograph of the patient shows extensive bilateral ossification between successive vertebral bodies with a bamboo spine appearance. Ankylosis of both the sacroiliac and hip joints is also seen. 


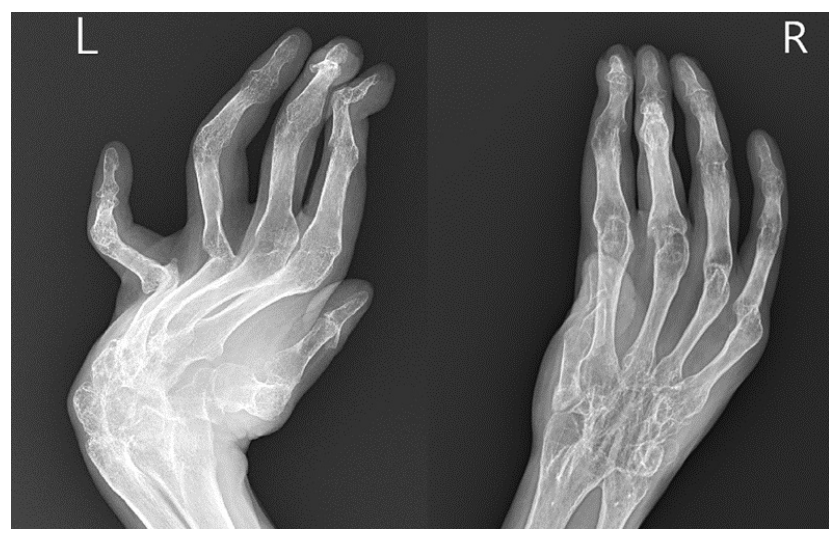

Figure 2. Plain radiographs of the patient show total ankylosis on the distal interphalangeal, proximal interphalangeal, metacarpophalangeal, carpometacarpal, intercarpal, and radiocarpal joints, except for the interphalangeal and metacarpophalangeal joints on the left thumb. Zig-zag deformity on the left fingers is also present. sinki principles and approved by the Soonchunhyang University Seoul Hospital Institutional Review Board (IRB: 2020-03-017). The need for informed consent was waived under the IRB authority.

\section{Conflict of interest}

No potential conflict of interest relevant to this article was reported. 